\title{
Risk factors for development of post-trabeculectomy endophthalmitis
}

Ordan J Lehmann, Catey Bunce, Melville M Matheson, Vincenzo Maurino, Peng T Khaw, Richard Wormald, Keith Barton

\begin{abstract}
Backgroundlaims-Although adjunctive use of antiproliferative agents improves the success rate of glaucoma filtration surgery it profoundly alters the morphology of the filtering bleb. In view of these structural changes, which have been suggested to predispose to bleb infection, the relative importance of potential risk factors in the development of posttrabeculectomy endophthalmitis was investigated.
\end{abstract}

Methods-A case-control study was performed on patients with posttrabeculectomy endophthalmitis presenting to a single academic centre over a $6^{1 / 2}$ year period. Cases were diagnosed by the combination of vitreous and aqueous inflammation occurring 4 or more weeks postoperatively with control patients chosen by selecting the three patients undergoing trabeculectomy immediately following each index case.

Results-Analysis of these data, derived from 23 cases and 69 controls, demonstrated that an episode of blebitis and the presence of diabetes mellitus were statistically significantly associated with subsequent endophthalmitis (odds ratios (OR) 11.8, 95\% CI: $2.21-88.31, p=0.003$ and $O R$ $4.51,95 \%$ CI $1.02-20.29, p=0.04$ respectively). The data also suggest an association exists between antiproliferative use and endophthalmitis (OR 3.3, 95\% CI $0.95-15.19, p=0.07)$ as the time interval between filtration surgery and development of endophthalmitis was significantly shorter in patients treated with antiproliferative agents $(p=0.001)$.

Conclusions-These results provide strong evidence of an increased risk of late endophthalmitis in patients who have diabetes mellitus or have had an episode of blebitis and suggest antiproliferative agents may also have an important role. (Br f Ophthalmol 2000;84:1349-1353)

Department of Pathology, Institute of Ophthalmology, University College, London

M M Matheson

Correspondence to: Mr O J Lehmann, Department of Molecular Genetics, Institute of

Ophthalmology, Bath Street, London EC1V 9EL, UK

OJLehmann@yahoo.com

Accepted for publication 23 May 2000

Late endophthalmitis is an infrequent potentially devastating complication of glaucoma filtering surgery. Not only may it occur in the unsuspecting patient many years after the procedure and progress with great rapidity, but the organisms responsible are often more virulent than those associated with post-cataract endophthalmitis. ${ }^{1}$

In the era of full thickness filtration procedures the reported rate of late endophthalmitis was up to $9 \%{ }^{2}$ and this was noted to decrease to $0.3 \%-1.5 \%$ following the introduction of partial thickness sclerostomies. ${ }^{34}$ The widespread introduction of antiproliferative agents such as 5-fluorouracil (5-FU) and mitomycin C (MMC) in the early 1990s, which result in thinner and less vascular filtering blebs, ${ }^{5}$ has led to concern that antiproliferatives may result in an epidemic of post-trabeculectomy endophthalmitis. ${ }^{6}$ Antiproliferative use certainly causes disordered conjunctival epithelial and stromal morphology, ${ }^{78}$ which is likely to reduce the filtering bleb's resistance to transconjunctival bacterial migration. ${ }^{3}$ However, the paucity of accurate incidence data for post-trabeculectomy endophthalmitis has hindered assessment of the risks of individual antiproliferatives.

While there is an increased risk of late endophthalmitis associated with inferiorly positioned trabeculectomies, ${ }^{9}{ }^{10}$ the risk associated with antiproliferatives applied to the superior conjunctiva is less clear cut. ${ }^{10-12}$ For this reason a case-control study was undertaken to determine the relative importance of potential risk factors, particularly antiproliferatives, in the development of post-trabeculectomy endophthalmitis at this academic centre based practice.

\section{Methods}

CASES AND CONTROLS

Patients presenting with post-trabeculectomy endophthalmitis to Moorfields Eye Hospital over a 6 $1 / 2$ year period (January 1993 to June 1999) were considered for inclusion. Patients treated during the period until December 1997 were identified retrospectively by cross referencing computerised records from the microbiological laboratory database with patients under the care of the glaucoma service clinics. (All cases of endophthalmitis have microbiological analysis and are included on this database.) In the subsequent 18 months (January 1998 to June 1999) cases were identified prospectively from those presenting to the glaucoma clinics. For each potential case the clinical records were reviewed to determine whether the treatment episode fulfilled the case definition for inclusion.

\section{CASE DEFINITION}

Late post-trabeculectomy endophthalmitis was diagnosed by the presence of vitreous inflammation together with a combination of bleb injection (or a bleb infiltrate) and anterior chamber inflammation ( $>2+$ of cells and flare) presenting 4 or more weeks after filtration surgery. Three control patients were chosen for 
Table 1 Relation between study factors and post-trabeculectomy endophthalmitis by case-control status

\begin{tabular}{|c|c|c|c|c|c|c|c|}
\hline \multirow[b]{2}{*}{ Study factor } & \multicolumn{2}{|c|}{ Cases $(n=23)$} & \multicolumn{2}{|c|}{ Controls $(n=69)$} & \multirow{2}{*}{$\begin{array}{l}\text { Unadjusted } \\
\text { odds ratio }\end{array}$} & \multirow[b]{2}{*}{$95 \% C I$} & \multirow[b]{2}{*}{$p$ Value ${ }^{\star}$} \\
\hline & No & $\%$ & No & $\%$ & & & \\
\hline Male & 15 & $(65)$ & 38 & (55) & 1.53 & $(0.57,4.25)$ & 0.47 \\
\hline Age $>60$ years & 11 & (50) & 40 & (58) & 0.73 & $(0.27,1.94)$ & 0.62 \\
\hline Right eye & 14 & (61) & 38 & (55) & 1.30 & $(0.48,3.44)$ & 0.81 \\
\hline Phakic & 19 & (83) & 62 & (90) & 0.54 & $(0.14,2.31)$ & 0.46 \\
\hline Fornix based flap & 9 & (39) & 31 & (45) & 0.79 & $(0.29,2.08)$ & 0.81 \\
\hline Releasable sutures & 14 & (61) & 45 & (65) & 0.83 & $(0.31,2.28)$ & 0.80 \\
\hline Diabetes mellitus & 5 & (22) & 4 & (6) & 4.51 & $(1.02,20.29)$ & 0.04 \\
\hline Bleb leak & 8 & (35) & 17 & $(25)$ & 1.63 & $(0.65,4.96)$ & 0.42 \\
\hline Blebitis & 6 & (26) & 2 & (3) & 11.82 & $(2.21,88.31)$ & 0.003 \\
\hline $\begin{array}{l}\text { Intraoperative } \\
\text { antiproliferative use }\end{array}$ & 20 & (87) & 46 & (67) & 3.33 & $(0.95,15.19)$ & 0.07 \\
\hline 5-Fluorouracil & 9 & (39) & 17 & (25) & 1.97 & $(0.70,5.37)$ & 0.19 \\
\hline$\beta$ Radiation & 2 & (9) & 5 & (7) & 1.22 & $(0.15,6.66)$ & 1.00 \\
\hline 0.1 Mitomycin & 2 & (9) & 7 & (10) & 0.84 & $(0.11,4.15)$ & 1.00 \\
\hline 0.2 Mitomycin & 3 & (13) & 11 & (16) & 0.79 & $(0.16,3.00)$ & 1.00 \\
\hline 0.4 Mitomycin & 4 & (17) & 6 & (9) & 2.21 & $(0.50,8.85)$ & 0.26 \\
\hline Total mitomycin & 9 & (39) & 24 & (35) & 1.21 & $(0.44,3.21)$ & 0.80 \\
\hline $\begin{array}{l}\text { Postoperative } \\
\text { subconjunctival 5-FU }\end{array}$ & 5 & (22) & 10 & (15) & 1.64 & $(0.45,5.40)$ & 0.52 \\
\hline
\end{tabular}

each endophthalmitis case by selecting from the operating theatre records the three patients undergoing trabeculectomy immediately following each index case. The study followed the tenets of the Declaration of Helsinki and was approved by the hospital's research ethics committee.

DATA COLLECTION

The data collected for each subject (summarised in Tables 1 and 2) included sex, age, diabetic status, and the nature of the filtration surgery (including technique employed, fornix/ limbal based conjunctival flap and, where relevant, dose, duration, and type of antiproliferative agent application). The postoperative interventions (including bleb needling and postoperative subconjunctival 5-FU injections) and the clinical features of the inflammatory episode (including Seidel positivity, grading of anterior chamber cellular reaction, presence of hypopyon, demonstration of vitritis with ultrasonography, and visual acuity) were recorded together with the time interval between surgery and the development of endophthalmitis. The occurrence of episodes of blebitis, defined as bleb infection in the absence of clinically apparent vitreous involvement, and whether this was concurrent with or preceded the endophthalmitis, was also noted. The number of patients undergoing trabeculectomy each calendar year was established from the hospital's computerised record of surgical activity in order to estimate the proportion of trabeculectomy cases that presented to Moorfields with subsequent endophthalmitis.

MICROBIOLOGICAL PROTOCOL

Anterior chamber and/or vitreous samples (as noted in Table 2) were processed according to a standard protocol. Each sample was inoculated on to blood, R2A, and Sabouraud's dextrose agar as solid phase media with cooked meat, brain-heart infusion, and thioglycolate broth used as enrichment media. Conjunctival cultures were not collected since organisms isolated from ocular surface swabs frequently differ from those cultured from intraocular samples. ${ }^{13}$

STATISTICAL ANALYSIS

Matching was employed in this study as a convenient method of choosing controls rather than as a means to control bias or increase precision of the odds ratios explored. However, since in general the analysis should reflect the design, we conducted both matched and unmatched analyses using respectively conditional logistic and logistic regression. ${ }^{14}$ Results were very similar, therefore we have presented only the simpler to interpret unmatched results - as odds ratios together with exact mid $\mathrm{p}$ confidence intervals and the $\mathrm{p}$ value of the Fisher's exact test of association. We did not compute adjusted odds ratios owing to the comparatively small data set and because the results of the univariate analyses did not suggest confounding. Analyses were conducted using STATACORP 97: Stata Statistical Software Release 5 (Stata Corporation, USA) and STATXACT version 2 (Cytel Software Corporation, Cambridge, MA, USA).

\section{Results}

Twenty six episodes of post-trabeculectomy endophthalmitis were identified (seven prospectively) of which three were excluded from analysis: two from patients who presented 5 and 14 days respectively following surgery and the other being the second occurrence of posttrabeculectomy endophthalmitis in the same patient. All cases had undergone glaucoma filtration surgery at Moorfields Eye Hospital. The characteristics of the 23 cases and 69 controls (Table 1) were similar with regard to a wide number of variables including age, sex, eye affected, phakic status and the surgical technique employed.

An episode of blebitis, which occurred in six of 23 cases (26\%) and two of 69 controls (3\%), was statistically significantly associated with post-trabeculectomy endophthalmitis; odds ratio 11.82 (95\% CI 2.21-88.31), p $=0.003$. 
Table 2 Clinical and microbiological features from 23 patients with post-trabeculectomy endophthalmitis

\begin{tabular}{|c|c|c|c|c|c|c|c|c|c|c|}
\hline $\begin{array}{l}\text { Glaucoma } \\
\text { type }\end{array}$ & $\begin{array}{l}\text { Time } \\
\text { interval }\end{array}$ & $\begin{array}{l}\text { Date of } \\
\text { presentation }\end{array}$ & $\begin{array}{l}\text { Antiproliferative } \\
\text { used }\end{array}$ & $\begin{array}{l}\text { Preop IOP } \\
\text { (mm } \mathrm{Hg})\end{array}$ & $\begin{array}{l}\text { Preop } \\
\text { medications }\end{array}$ & $\begin{array}{l}\text { IOP at last } \\
\text { follow up } \\
\text { (mm Hg) }\end{array}$ & $\begin{array}{l}\text { Medications } \\
\text { at last follow } \\
\text { up }\end{array}$ & Organism isolated & $\begin{array}{l}\text { Decrease in } \\
\text { acuity } \\
\text { (Snellen } \\
\text { lines) }\end{array}$ & $\begin{array}{l}\text { Acuity at } \\
\text { last follow } \\
\text { up }\end{array}$ \\
\hline POAG & 6 years & 1999 & 5-Fluorouracil & 27 & 1 & 14 & 1 & Moraxella & 0 & Sn $6 / 9$ \\
\hline POAG & 14 years & 1999 & None & 24 & 2 & 22 & 3 & & 2 & Sn $6 / 18$ \\
\hline POAG & 4 years & $1999^{\circ}$ ब & 5-Fluorouracil & 28 & 3 & 9 & & & 1 & PL \\
\hline POAG & 6 years & 1999 & None & 35 & 4 & 10 & & & 1 & Sn $6 / 12$ \\
\hline POAG & 1 month & 1998 & Mitomycin (0.2) & 24 & 3 & 10 & & $S$ viridans & 6 & $\mathrm{CF}$ \\
\hline POAG & 4 months & $1998^{\circ}$ & 5-Fluorouracil & 25 & & 12 & & & 4 & Sn $6 / 24$ \\
\hline Congenital & 3 years & $1998 \ddagger$ & Mitomycin (0.4) & 33 & 2 & 10 & & Gram -ve bacillus $†$ & 3 & $\mathrm{CF}$ \\
\hline AACG & 7 months & 1998 & 5-Fluorouracil & 22 & 3 & 12 & & & 3 & Sn $6 / 36$ \\
\hline POAG & $\begin{array}{l}15 \\
\text { months }\end{array}$ & $1998 \ddagger^{\circ}$ & 5 Fluorouracil & 26 & 1 & 4 & & $S$ pneumoniae & 5 & $\mathrm{HM}$ \\
\hline POAG & 1 month & $1998 \ddagger^{\circ}$ & Mitomycin (0.1) & 18 & 1 & 16 & 1 & $S$ p [neumoniae & 9 & PL \\
\hline NTG & 6 months & 1998 & Mitomycin (0.1) & 16 & & 12 & & & 3 & Sn $6 / 24$ \\
\hline POAG & 1 month & $1997 \ddagger \Omega$ & 5-Fluorouracil & 25 & 3 & 21 & 3 & Gram -ve bacillus $†$ & 0 & Sn $6 / 24$ \\
\hline POAG & 3 months & 1997 & 5-Fluorouracil & 24 & 2 & 12 & & & 0 & Sn $6 / 9$ \\
\hline POAG & 1 month & 1997 & Mitomycin (0.2) & 22 & 2 & 21 & 2 & & 1 & Sn $6 / 12$ \\
\hline POAG & 4 years & 1997 & Mitomycin (0.2) & 22 & 3 & 3 & & $\star$ & 0 & HM \\
\hline Congenital & 9 years & 1997 & $\beta$ radiation & 30 & & 20 & 1 & H influenzae & 1 & HM \\
\hline Traumatic & 15 years & 1996 & $\begin{array}{l}\text { None (bleb } \\
\text { revision) }\end{array}$ & 36 & & 13 & & & 0 & Sn $6 / 9$ \\
\hline POAG & 1 month & $1996^{\circ}$ ब & 5-Fluorouracil & 26 & 2 & 18 & 2 & $S$ aureus & 0 & Sn $6 / 9$ \\
\hline POAG & 2 years & 1996 『 & Mitomycin (0.4) & 24 & 3 & 10 & & $\begin{array}{l}\alpha \text { hamolytic } \\
\text { streptococcus }\end{array}$ & 8 & PL \\
\hline Congenital & 4 months & $1995 \ddagger$ & Mitomycin (0.4) & 34 & & 16 & 4 & & 1 & $\mathrm{CF}$ \\
\hline Congenital & 4 years & $1995 \ddagger^{\circ}$ & Mitomycin (0.4) & 46 & 2 & 30 & 3 & $S$ albus & 1 & PL \\
\hline POAG & 1 month & $1994 \ddagger$ & 5-Fluorouracil & 26 & 2 & 18 & 2 & & 0 & Sn $6 / 9$ \\
\hline Congenital & 6 years & 1993 & $\beta$ radiation & 28 & 1 & Phthisis & & & 2 & NPL \\
\hline
\end{tabular}

POAG = primary open angle glaucoma; Congenital = congenital glaucoma; AACG = acute angle closure glaucoma; NTG = normal tension glaucoma; Time inter$\mathrm{val}=$ interval between filtration surgery and episode of post-trabeculectomy endophthalmitis in whole months or years; 1999 refers to the first 6 months of $1999 ; \beta$ radiation applied at a dosage of $750 \mathrm{cGy}$; Preoperative IOP = IOP before filtering surgery; ${ }^{\star}$ no vitreous tap performed (all other cases treated with intravitreal vancomycin $(2 \mathrm{mg})$ and amikacin $(0.4 \mathrm{mg})$; †demonstrated with light microscopy, no growth on culture media; fultrasound confirmation of vitreous involvement; §denotes bleb needled postoperatively; ${ }^{\circ}$ denotes previous episode of blebitis; $₫$ denotes bleb leak. All antiproliferative agents were applied for 5 minutes and the dose of mitomycin $\mathrm{C}$ is shown in parentheses. No patients were prescribed topical antibiotics for more than 3 weeks following trabeculectomy surgery.

The mean time interval between these treated episodes of blebitis and the subsequent development of endophthalmitis was 9 weeks (SD 4.2). Diabetes mellitus occurred more commonly in cases than controls (five of $23(22 \%)$ versus four of $69(6 \%)$ ) and was also statistically significantly associated with post-trabeculectomy endophthalmitis; odds ratio 4.51 (95\% CI 1.0220.29), $p=0.04$. Antiproliferatives were widely used intraoperatively with mitomycin $\mathrm{C}$ being applied most frequently in both cases and controls. The time interval between filtration surgery and development of endophthalmitis was significantly shorter in antiproliferative treated cases ( $p=0.001$, rank sum test). Use of an antiproliferative agent (20 of 23 cases $(87 \%)$ versus 46 of 69 controls $(67 \%)$ ) was associated with subsequent endophthalmitis with an odds ratio of 3.33 (95\% CI 0.95-15.19), although these results did not reach statistical significance, $\mathrm{p}=0.07$.

The clinical characteristics of the 23 patients diagnosed with post-trabeculectomy endophthalmitis and the organisms isolated in 10 cases $(43 \%)$ are summarised in Table 2 . The median time interval between glaucoma surgery and endophthalmitis was 15 months (range 1-180) and the mean reduction in acuity following the episode of endophthalmitis was two Snellen lines (SD 2.6). At the latest follow up (median 7 months, range 1-154) 10 of 23 eyes (43\%) required medication to control the intraocular pressure and a further three eyes (13\%) were either hypotonous or phthisical.

\section{Discussion}

Case-control methodology was applied to patients presenting with post-trabeculectomy endophthalmitis to a single hospital over a $61 / 2$ year period in order to clarify the risk factors for developing the condition.

The results at the univariate level provide strong evidence that the odds of endophthalmitis are increased approximately 12-fold (OR 11.82, 95\% CI 2.21-88.31, p = 0.003) in a patient in whom blebitis has occurred. This result is in keeping with the recognised ability of blebitis to progress to endophthalmitis ${ }^{15}$ and it is likely that the changes in bleb morphology that predispose to bleb related infection increase susceptibility for subsequent endophthalmitis. Although the confidence interval associated with the odds ratio is wide, illustrating the imprecise nature of this estimate, even the lower confidence limit lies well above unity. These data also provide evidence that endophthalmitis is more likely in a patient who has diabetes mellitus (OR 4.51, 95\% CI 1.02$20.29, \mathrm{p}=0.04)$; a finding in keeping with diabetes being a proved risk factor for other types of endophthalmitis. ${ }^{16}{ }^{17}$

Although the power of this study was limited-a $57 \%$ chance of detecting an odds ratio of 5 (assuming $\alpha=0.05$, three controls per case and $70 \%$ exposure among controls) these data provide some evidence that the odds of endophthalmitis may be greater in patients in whom antiproliferatives are used $(p=0.07)$. A patient treated with antiproliferatives is estimated to be three times as likely to develop endophthalmitis as one who is not; however, the $95 \%$ confidence interval is just consistent with there being no difference in odds. An adjusted odds ratio was not computed because of the limited data set and because of concerns that blebitis may lie along a postulated causal 
path between antiproliferative use and endophthalmitis. An association was also found between antiproliferative use and a shorter time interval to the development of endophthalmitis $(\mathrm{p}=0.001)$. This result may, however, partly reflect ascertainment bias as the study included patients developing endophthalmitis within a $6 \frac{1}{2}$ year period (patients undergoing trabeculectomy in the era before widespread antiproliferative agent use could have developed endophthalmitis within a year or two of surgery without being ascertained). Other factors were not found to be associated with endophthalmitis.

These results indicate that blebitis, diabetes mellitus and, possibly, antiproliferative use are associated with subsequent endophthalmitis. Migration of bacteria across the filtering bleb is recognised to be the initial step in the development of blebitis ${ }^{318}$ and ordinarily such spread is prevented by the physical properties of the bleb together with the eye's innate immune response. Antiproliferative use alters the thickness, cellularity, and vascularity of the filtering bleb's conjunctiva. The thinner and relatively avascular blebs that result frequently have areas of absent conjunctival epithelium which are demonstrable both microscopically ${ }^{8}$ and clinically. These areas of denuded epithelium and the underlying full thickness stromal damage ${ }^{7}$ compromise the bleb's physical barrier function and affect the conjunctival contribution to the ocular innate immune response. One component of this response is a family of highly conserved cysteine rich antimicrobial peptides, called defensins, that combat infection at a wide range of mucosal surfaces..$^{19} 20$ The constitutive conjunctival expression of certain defensin isoforms $\left(\beta_{1}\right)$ at a high level ${ }^{21}$ suggests that they are important components of the conjunctiva's innate defences. $\beta$ Defensin expression will be altered in unepithelialised areas of the filtering bleb and we hypothesise that the combination of breaches in the bleb's barrier function with altered conjunctival $\beta$ defensin production may account for part of the observed increased susceptibility to infection of antiproliferative agent treated blebs.

Direct comparison between published reports of post-trabeculectomy endophthalmitis cases is hampered by variation between studies regarding factors including study design, case definition, case mix, antiproliferative agent use (both type, dose and duration of administration), as well as the nature and site of surgery (superior/ inferior filtration) and the treatment received on presentation with endophthalmitis. ${ }^{19-11} 1522-25$ For these reasons we have limited comparison of the clinical features (Table 2) of this group of patients to noting that the visual outcome and rate of filtering bleb survival are broadly comparable with previous reports (and in a study period partially predating the Endophthalmitis Vitrectomy Study ${ }^{26}$ that 22 of the 23 cases received intravitreal antibiotics). The poor prognosis associated with post-trabeculectomy endophthalmitis is best highlighted by the fact that just three of 23 eyes achieved satisfactory intraocular pressures without treatment and a Snellen acuity greater than $6 / 24$. Overall epi- sodes of post-trabeculectomy endophthalmitis were associated with a high likelihood of trabeculectomy failure (57\%) and a mean reduction in acuity of two Snellen lines. This measurement understates the effect of visual loss in individual patients with poor initial acuity as a result of advanced or congenital glaucoma and the number of patients in the latter subgroup (five of 23) may skew the overall visual results reported. The proportion of cases $(52 \%)$ occurring more than 1 year postoperatively with some occurring several years later indicates that the risk of post-trabeculectomy endophthalmitis is long lasting, perhaps for at least the lifetime of a functioning bleb. ${ }^{6}$ This is of particular concern in younger glaucoma patients in whom surgery is the main treatment modality. The design of the study precluded calculation of incidence data owing to the likelihood of acertainment bias and the study may have been biased to detecting fewer cases of late endophthalmitis than if all trabeculectomy cases had been followed prospectively. None the less the rise in the number of cases presenting to Moorfields is compatible with reports ${ }^{10}{ }^{11}$ suggesting that the frequency of post-trabeculectomy endophthalmitis is increasing. Whether the more favourable bleb morphology achieved with larger areas of antiproliferative treatment ${ }^{27}$ has the potential for reducing the frequency of this complication remains to be determined.

Proprietary interest: None

We are grateful to Mr R A Hitchings for his advice and support and to Miss Tringham for providing data on the hospital's surgical activity.

1 Mandelbaum S, Forster RK, Gelender H, et al. Late onset endophthalmitis associated with filtering blebs. Ophthalmology 1985;92:964-72.

2 Sugar SH, Zekman T. Late infection of conjunctival filtering scars. Am f Ophthalmol 1958;46:155-70.

3 Katz LJ, Cantor LB, Spaeth GL. Complications of surgery in glaucoma. Ophthalmology 1985;92:959-63.

4 Wilson P. Trabeculectomy: long-term follow-up. $\mathrm{Br} \mathcal{F}$ Ophthalmol 1977;61:535-8.

5 Kitazawa Y, Kawase K, Matsushita H, et al. Trabeculectomy with mitomycin. A comparative study with fluorouracil. Arch Ophthalmol 1991;109:1693-8.

6 Parrish R, Minckler D. "Late endophthalmitis"-filtering Parrish R, Minckler D. "Late endophthalmitis"- - filte
surgery time bomb? Ophthalmology 1996;103:1167-8.

7 Shields B, Scroggs MW, Sloop CM, et al. Clinical and Shields B, Scroggs MW, Sloop CM, et al. Clinical and
histopathological observations concerning hypotony after trabeculectomy with mitomycin C. Am F Ophthalmol 1993; 116:673-83

8 Nuyts RMMA, Felten PC, Pels E, et al. Histopathological effects of mitomycin $\mathrm{C}$ after trabeculectomy in human glaucomatous eyes with persistent hypotony. $A m$ f Ophthalmol 1994;118:225-37.

9 Caronia RM, Liebmann JM, Friedman R, et al. Trabeculectomy at the inferior limbus. Arch Ophthalmol 1996;114: $387-91$.

10 Higginbotham EJ, Stevens RK, Musch DC, et al. Blebrelated endophthalmitis after trabeculectomy with mitomycin C. Ophthalmology 1996;103:650-6.

11 Greenfield DS, Suñer IJ, Miller MP, et al. Endophthalmitis after filtering surgery with mitomycin. Arch Ophthalmol 1996;114:943-9.

12 Mochizuki K, Jikihara S, Ando Y, et al. Incidence of delayed onset infection after trabeculectomy with adjunctive mitomycin C or 5-fluorouracil treatment. Br F Ophthalmol 1997;81:877-83.

13 The Fluoruracil Filtering Surgery Group. Five year follow-up of the fluorouracil filtering surgery study. $A m \mathcal{F}$ Ophthalmol 1996;121:349-66.

14 Altman DG. Practical statistics for medical research. 1st ed. London: Chapman and Hall, 1991:351-8.

16 Ciulla TA, Beck AD, Topping TM, et al. Blebitis, early endophthalmitis, and late endophthalmitis after glaucomafiltering surgery. Ophthalmology 1997;104:986-95.

16 Phillips WB, Tasman WS. Postoperative endophthalmitis in Phillips WB, Tasman WS. Postoperative endophthalmitis in
association with diabetes mellitus. Ophthalmology 1994; 101:508-18.

17 Kattan HM, Flynn HW, Pflugfelder SC, et al. Nosocomial endophthalmitis survey:current incidence of infection after intraocular surgery. Ophthalmology 1991;98:227-38. 
18 Brown RH, Yang LH, Walker SD, et al. Treatment of bleb infection after glaucoma surgery. Arch Ophthalmol 1994, 112:57-61.

19 Hancock REW. Peptide antibiotics. Lancet 1997;349:41822.

20 Haynes RJ, Tighe PJ, Dua HS. Innate defence of the eye by anti-microbial defensin peptides. Lanct 1998:352:451-2.

21 Lehmann OJ, Hussain IR, Watt PJ. Investigation of $\beta$-defensin gene expression in the ocular anterior segment by semiquantitative PCR. Br f Ophthalmol (in press).

22 Kangas TA, Greenfield DS, Flynn HW, et al. Delayed-onset endophthalmitis associated with conjunctival filtering blebs. Ophthalmology 1997;104:746-52.

23 Wolner B, Liebmann JM, Sassani JW, et al. Late bleb-related endophthalmitis after trabeculectomy with adiunctive 5-fluorouracil. Ophthalmology 1991;98:1053-60.
24 Phillips WB, Friedberg MA, Wong TP, et al. Late onset endophthalmitis associated with filtering blebs. Ophthalmic Surg 1994;25:88-91.

25 Waheed S, Ritterband DC, Greenfield DS, et al. Blebrelated ocular infection in children after trabeculectomy with mitomycin C. Ophthalmology 1997;104:2117-20.

26 Endophthalmitis Vitrectomy Study Group. Results of the Endophthalmitis Vitrectomy Study. A randomized trial of immediate vitrectomy and of intravenous antibiotics for the treatment of postoperative bacterial endophthalmitis. Arch Ophthalmol 1995;113:1479-96.

27 Cordeiro MF, Constable PH, Alexander RA, et al. Effect of varying the mitomycin $\mathrm{C}$ treatment area in glaucoma filtration surgery in the rabbit. Invest Ophthalmol Vis Sci 1997;38:1639-46. 